\title{
USING THE LANE-CHANGE TEST (LCT) TO ASSESS DISTRACTION: TESTS OF VISUAL-MANUAL AND SPEECH-BASED OPERATION OF NAVIGATION SYSTEM INTERFACES
}

\author{
Joanne L. Harbluk, Peter C. Burns, Martin Lochner, \& Patricia L. Trbovich \\ Transport Canada \\ Ottawa, Ontario, Canada, K1A 0N5 \\ E-mail: harbluj@tc.gc.ca
}

\begin{abstract}
Summary: The Lane Change Test (LCT) is an easy-to-implement, low-cost methodology for the evaluation of the distraction associated with performing invehicle tasks while driving (Mattes, 2003). In the present study, the LCT was used to assess driving performance when drivers completed navigation tasks using visual-manual or speech-based interfaces. Drivers performed two types of navigation tasks at two levels of difficulty. The results provide support for the LCT as an effective measure of distraction for both types of interface. It is recommended that the LCT procedure incorporate additional measures beyond the current mean deviation measure. Two measures are suggested: Lane Change Initiation, which reflects the aspects of driving having to do with detection and response delay as a result of distraction, and a measure of task duration to account for risk exposure.
\end{abstract}

\section{INTRODUCTION}

The purpose of telematics devices is to deliver information to drivers. These in-vehicle technologies provide drivers with navigation guidance, information about the road and traffic conditions, as well as other types of information such as entertainment and communication via cell or web-based applications. The common element across all these examples, regardless of information content, is that drivers must interact with these systems to obtain the desired information; consequently, these in-vehicle devices have the potential to distract as well as inform.

Telematics devices are increasingly popular with drivers and offer an ever-expanding choice of applications. Affordable hardware and increased volume have made telematics systems more affordable for consumers and OEMs (ThomasNet, 2006). Driver interaction with these in-vehicle technologies, however, represents a potentially serious road safety threat. It is essential that these in-vehicle devices be designed to minimize this risk in order to ensure the safety of drivers and other road users.

Appropriate protocols are required for assessing the distraction potential of in-vehicle tasks and devices. These methodologies must be valid, objective and reliable. In addition, they must be low cost and practical if they are to be implemented. Ideally, a test or suite of tests that meet these requirements could be standardized and made widely available. Considerable research activity has been concentrated on this effort in the form of a number of large-scale projects (e.g., ADAM, AIDE, CAMP, HASTE), as well as symposia and workshops such as the recent Driver Metrics Workshop (2006). 
One assessment methodology, the Lane Change Test (LCT), has received considerable attention since it appears to be a practical and effective measure of the costs associated with operating invehicle devices while performing a driving task. The LCT was a product of the ADAM (Advanced Driver Attention Metrics) project (Mattes, 2003) and is appealing for a number of reasons. It is easy to implement, standardized, low cost and, unlike other methods such as Occlusion, it is not restricted to the assessment of visual-manual distraction. The procedure is currently under ISO development (ISO, 2006).

The procedure requires a minimum amount of equipment and is completed quickly. Drivers are seated at a desktop computer and are required to repeatedly perform lane changes when prompted by road signs. The quality of these lane changes provides the metric for comparison. Lane change performance under dual task conditions (driving and using the telematics system of interest) is evaluated against a normative model of single task performance. The difference between the driver's actual lane change and the ideal lane change path is influenced by the drivers' performance for detecting and responding to the road signs as well as their lateral control maintenance. The extent to which the dual task condition results in increased distraction is reflected in impaired lateral control and event detection.

The purpose of the present study was to further explore the LCT with a particular focus on its efficacy as a procedure for assessing the cognitive distraction associated with the use of speechbased interfaces as well as visual-manual interfaces. A navigation system designed for use in both modes was obtained from a currently available vehicle. The task of interacting with the navigation system was selected since programming a destination into a navigation system has been reported to be one of the most complex tasks carried out on a telematics device (Llaneras \& Singer, 2002). We also wanted to explore the utility of an additional measure of driver performance, one that closely reflects detection performance in the driving environment in addition to the typical measure that captures mean deviation in the lane change path. This measure is known as Lane Change Initiation, and it reflects the delay in the initiation of the required lane change manoeuvre as function of performing the additional task while driving. Finally, because risk exposure is a function of secondary task duration as well as intensity, frequency and timing, we include a measure of the individual task durations.

\section{METHOD}

\section{Participants}

Participants using the visual-manual interface ranged in age from 24 to 58 years with a mean age of 34. Participants using the speech-based interface ranged in age from 21 to 48 years with a mean age of 33. Sixteen participants ( 13 men; 3 women) took part in each of the interface sessions. All drivers were fully licensed and drove a minimum of $10 \mathrm{~K}$ kilometres annually.

\section{Equipment \& Materials}

LCT. A PC was used to present the "lane change test" driving simulation on a video monitor. A Logitech MOMO Racing Force-Feedback Wheel with foot pedals was used for the control of the simulated vehicle (Figure 1). The test track was a $3 \mathrm{~km}$ straight lane road with 18 lane change 
signs. The mean distance between signs was $150 \mathrm{~m}$, resulting in a mean duration of 9 seconds between two lane changes, since participants were required to perform lane-change manoeuvres while maintaining a constant speed of $60 \mathrm{~km} /$ hour (resulting in duration of approximately 180 seconds per track).

The top frame of Figure 2 shows the driver's view of the simulated three-lane road scene with signs instructing the driver to change into the left lane. The middle frame illustrates the difference in area (black) between the normative path and actual path for a quick response (adapted from Mattes, 2003). The bottom frame illustrates performance during a slower lanechange response where the greater black area indicates poorer performance.

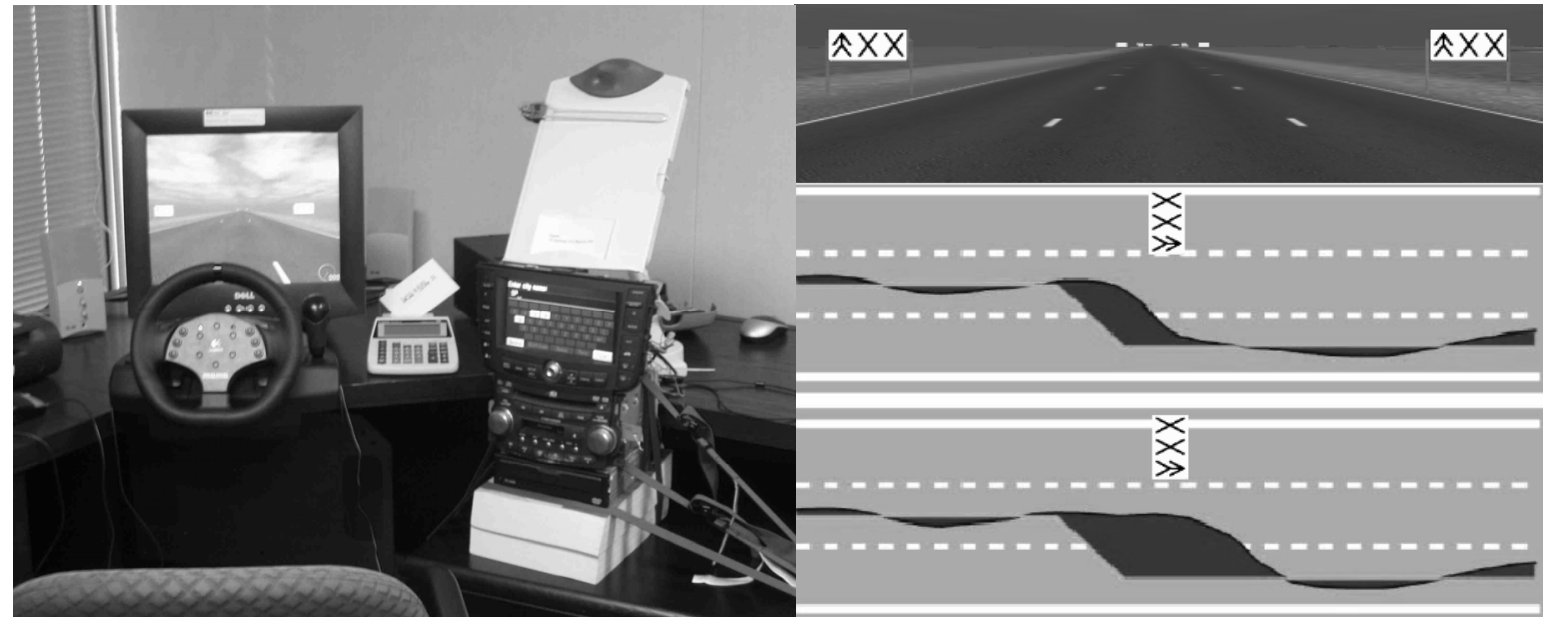

Figure 1. LCT Equipment Set Up

Figure 2. Image of LCT monitor view and illustrations of performance.

Navigation system. The navigation system, acquired from a 2005 ACURA-TL, could be operated in visual manual or speech mode. In visual-manual mode, drivers made selections using the touch screen. In speech mode, drivers used voice control to select their choices using single word commands.

Tasks. Two typical navigation system tasks, Point of Interest (POI) Entry and Address Entry, were used in the study. These particular tasks were chosen from among the HASTE tasks (Johansson, et al., 2004) because they are real-world navigation tasks which have manipulated levels of complexity. Each task type had two levels of complexity differentiated by the amount of information the driver was required to input into the system.

\section{Procedure}

Participants were instructed to perform their lane change manoeuvres as soon as they could recognize the information on the sign. They were instructed to change lanes in a deliberate manner, as quickly and efficiently as possible. After receiving instructions, participants began the session with practice trials on the LCT with and without secondary tasks. Participants then completed one 3-minute run for each of the 4 experimental conditions, as well as for the three 
baseline conditions. Participants drove the track without performing a secondary task during baseline drives. For the secondary task drives, participants drove while performing Point of Interest (POI) and Address Entry tasks, each with a low and high level of complexity. The information for the secondary tasks was provided on $3 \times 5$ cards. The order of the experimental conditions was counterbalanced. Participants were reminded that their primary task was to drive as safely as possible, as they would in the real world, and were asked to perform secondary tasks as quickly and accurately as possible. There was no surrounding traffic in the scenario.

\section{RESULTS}

Three measures of performance were calculated and analyzed:

- Mean deviation in lane change path (MDEV): mean deviation between the position of the normative model and the actual driven course.

- Lane change initiation (LCI): the difference (in metres) between the distance at which the lane change direction appears on a sign (i.e., distance at which the driver would ideally initiate a lane change) and the distance at which the driver actually initiated the lane change. In the present experiment, lane change initiation represents an event detection measure.

- $\quad$ Task duration: the mean time (in seconds) taken to complete a secondary task while driving. The duration required to complete a specific task is an important measure as it indicates the amount of time the driver's attention will be drawn away from the primary task of driving.

Table 1. Results for the Manual Interface

\begin{tabular}{|l|r|r|r|}
\hline \multicolumn{4}{|l|}{ MANUAL INTERFACE } \\
\hline Tasks & \multicolumn{1}{l|}{ MDEV } & \multicolumn{1}{l|}{ LCI } & Duration \\
\hline Baseline & 0.85 & 12.07 & \multicolumn{1}{c|}{} \\
\hline Lo POI & 1.07 & 13.30 & 5.51 \\
\hline Lo Address & 1.06 & 14.67 & 6.16 \\
\hline Hi POI & 1.21 & 16.13 & 37.26 \\
\hline Hi Address & 1.32 & 16.99 & 45.17 \\
\hline
\end{tabular}

Table 2. Results for the Speech Interface

\begin{tabular}{|l|r|r|c|}
\hline \multicolumn{4}{|l|}{ SPEECH INTERFACE } \\
\hline Tasks & \multicolumn{1}{l|}{ MDEV } & \multicolumn{1}{l|}{ LCI } & Duration \\
\hline Baseline & 0.96 & 12.45 & - \\
\hline Lo POI & 1.16 & 15.89 & 32.25 \\
\hline Lo Address & 1.10 & 15.96 & 38.61 \\
\hline Hi POI & 1.16 & 16.83 & 105.80 \\
\hline Hi Address & 1.15 & 16.44 & 102.94 \\
\hline
\end{tabular}

(Different shades indicate statistically significant differences at $\mathrm{p}<.05$ )

Mean deviation in lane change path. Analyses of data from the manual interface conditions indicated that deviations exceeded baseline for all four task conditions $\left(\mathrm{F}_{(4,60)}=10.81, \mathrm{p}<.001\right.$; all conditions greater than baseline at $\mathrm{p}<.001)$. Within the task conditions, there was a main effect of task complexity $\left(\mathrm{F}_{(1,15)}=14.39, \mathrm{p}<.001\right)$, where more complex tasks produced greater mean deviations. 
Analyses for the speech interface conditions revealed a similar pattern where all task conditions exceeded the baseline values $\left(\mathrm{F}_{(4,60)}=5.47, \mathrm{p}<.001\right.$; all conditions greater than baseline $\left.\mathrm{p}<.01\right)$. However, there were no additional effects of task type or complexity as were seen in the manual interface conditions (ps>.05).

Lane change initiation. The analyses for the manual interface data revealed that drivers took longer to initiate a lane change when engaged in any task in addition to driving $\left(F_{(4,60)}=19.16\right.$, $\mathrm{p}<.001$; all individual conditions greater than baseline $\mathrm{p}<.05$ ). Examination of the specific task conditions indicated that drivers took longest in the more demanding Hi Complexity conditions than the Low Complexity conditions $\left(\mathrm{F}_{(1,15)}=21.70, \mathrm{p}<.001\right)$. There was also a significant effect of Task Type $\left(\mathrm{F}_{(1,15)}=5.07, \mathrm{p}<.05\right)$ indicating that drivers took longer to initiate the lane change when engaged in the Address tasks than the POI tasks.

The analyses for the speech interface lane change initiation data indicated that when engaged in any task, the drivers took longer to initiate their lane changes $\left(F_{(4,60)}=7.00, p<.001\right)$. There were no differences among the various task conditions although all were greater than the baseline $(\mathrm{p}<.001)$.

Task Duration. For the manual interface, the main effect for Task complexity indicated that the more complex tasks clearly took more time than the less complex tasks $\left(\mathrm{F}_{(1,15)}=315.41, \mathrm{p}<.001\right)$. There was also a marginal effect of task type $\left(\mathrm{F}_{(1,15)}=4.31, \mathrm{p}<.055\right)$, reflecting that the address tasks took longer than the POI tasks.

The analyses of Task Duration for the speech interface confirmed that the Hi Complexity tasks took considerably longer than the Low complexity tasks $\left(\mathrm{F}_{(1,15)}=72.62, \mathrm{p}<.001\right)$.

\section{DISCUSSION}

In this study, both measures calculated from the LCT, mean deviation in lane change path (MDEV) and lane change initiation (LCI), reflected the costs associated with performing secondary tasks while driving. These findings held for all secondary tasks and both interface types. Participants demonstrated greater mean deviation in lane change path and took longer to initiate their lane changes when they were engaged in secondary tasks compared with baseline driving. For the manual interface, the MDEV measure was sensitive to task complexity. The lane change initiation measure reflected a similar pattern in discriminating among the tasks and, in addition, it distinguished between the two task types at the low level of complexity. For the speech interface, both MDEV and LCI were greater from baseline for all tasks but did not reflect any differences across the tasks themselves.

The proscribed 180s duration of the LCT procedure results in a situation where shorter tasks may be repeated several times during the course of the testing procedure while longer tasks may be completed only once. Because the MDEV measure is the average instantaneous deviation from the ideal path, there is no accounting for the cumulative exposure risk, which increases as a function of task duration. Mean durations for tasks in the present study ranged from approximately 5 to 105 seconds. Task duration must be included in an evaluation of safety 
assessment of interaction with telematics devices. Additional work is required to identify procedures on how best to accomplish this.

Although speech-based interfaces are increasingly popular choices for in-vehicle technologies, there are likely to be advantages and disadvantages associated with their use in different applications (e.g., Tsimhoni et al., 2004). The present results provide support for the use of the LCT in the evaluation of speech-based, as well as manual, interfaces, a definite advantage for LCT over the Occlusion method, which is narrower in scope.

In summary, the present results provide support for the LCT as an effective measure of distraction. There is, however, a need for measures beyond the current MDEV (e.g., Burns et al., 2005; Trbovich, 2006). The LCI measure provides a useful addition to the MDEV metric as it reflects the aspects of driving having to do with detection and delay, aspects of driving with known safety relevance in the real world. The current ISO document would benefit from the addition of this measure as well as a measure incorporating the impact of task duration. Although outside the scope of the ISO document, safety performance criteria will need to be established to make the LCT a useful tool for the assessment of distraction associated with the use in-vehicle technologies.

\section{ACKNOWLEDGMENTS}

We would like to thank Denis Boucher for his work on the Lane Change Initiation Measure.

\section{REFERENCES}

Burns, P.C., Trbovich, P.L., McCurdie, T., \& Harbluk, J.L. (2005). Measuring Distraction: Task Duration and the Lane-Change Test (LCT). Proceedings of the $49^{\text {th }}$ Annual Meeting of the Human Factors and Ergonomics Society.

Driver Metrics Workshop. (2006). Ottawa, Canada, October 2006. Presentations available at http://ppc.uiowa.edu/drivermetricsworkshop

ThomasNet. (2006). US Car Telematics Devices Market Explodes After Years of Stagnation. http://news.thomasnet.com/companystory/480360

ISO Committee Draft. (2006). Road vehicles_-Ergonomic aspects of transport information and control systems - Simulated lane change test to assess driver distraction. ISO TC 22/SC 13 N WG8 N417.

Johansson, E., Carsten, O., Janssen, W., Jamson, H., Jamson, S., Ostlund, J., Brouwer, R., Mouta, S., Harbluk, J., Antilla, V., Sandberg, H., \& Luoma, J. (2004). HASTE Deliverable 3: Validation of the HASTE protocol specification. http://www.its.leeds.ac.uk/projects/haste/downloads/Haste_D3.pdf

Llaneras, R.E. \& Singer, J.P. (2002). Inventory of In-Vehicle Technology Human Factors Design Characteristics. National Highway Traffic Safety Administration Report No. DOT HS 809 457; Washington, DC. 
Mattes, S. (2003). The Lane Change Task as a Tool for driver Distraction Evaluation. IHRA-ITS Workshop on Driving Simulator Scenarios, October 2003 - Dearborn, Michigan. www.nrd.nhtsa.dot.gov/IHRA/ITS/MATTES.pdf

Trbovich, P.L. (2006). Effects of phonological, visual and spatial information processing on a simulated driving task. Unpublished doctoral thesis, Carleton University, Ottawa, ON.

Tsimhoni, O., Smith, D., \& Green, P. (2004). Address entry while driving: Speech recognition versus a touch-screen keyboard. Human Factors, 46, 600-610. 